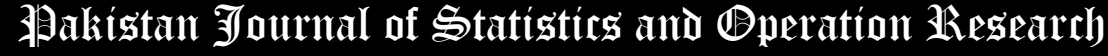

\section{Characterizations of Discrete Weibull Distributions}

\author{
G.G. Hamedani ${ }^{1 *}$, Nadeem Shafique Butt ${ }^{2}$
}

* Corresponding Author

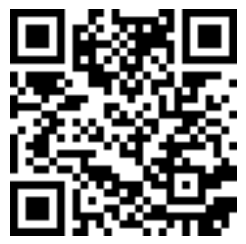

1. Department of Mathematical and Statistical Sciences Marquette University Milwaukee, WI 53201-188, gholamhoss.hamedani@marquette.edu

2. Department of Family and Community Medicine, King Abdulaziz University, Saudi Arabia, nshafique@kau.edu.s

\begin{abstract}
Seven versions of the discrete Weibull distribution are characterized via conditional expectation of function of the random variable as well as based on the hazard or reverse hazard function.
\end{abstract}

Key Words: Discrete Weibull distribution, Discrete Modified Weibull distribution, Discrete Reduced Modified Weibull distribution, Characterizations.

Mathematical Subject Classification: 60E10, 60E15.

\section{Introduction}

As mentioned in our previous works, the problem of characterizing a distribution is an important problem in applied sciences, where an investigator is vitally interested to know if their model follows the right distribution. To this end the investigator relies on conditions under which their model would follow specifically chosen distribution. In this work, we present certain characterizations of the discrete Weibull distributions listed above, which have been taken from the interesting review paper of Almalki and Nadarajah (2014). These characterizations are based on: ( $i$ ) conditional expectation of certain functions of the random variables; ( $i i)$ the hazard function and ( $i i i)$ the inverse hazard function. In what follows we use the same notation for the parameters as used by the original authors. For a detailed treatment of each one of these distributions and their domain of applicability, we refer the interested reader to the corresponding paper cited in the references. We certainly hope that the contents of this work will be useful to a good number of researchers whose models follow discrete distributions.

\subsection{Preliminaries}

The cumulative distribution function (cdf), the corresponding probability mass function (pmf), the hazard function (hf) and the reverse hazard function (rhf) of each of the distributions mentioned above, are listed below in $(a)-(f)$.

(a) The cdf, pmf, hf and rhf of DWI distribution are given, respectively, by

$$
\begin{aligned}
& F(x)=F(x ; \theta, q)=1-q^{(x+1)^{\theta}}, \quad x \in \mathbb{N}^{*}=\mathbb{N} \cup\{0\}, \\
& f(x)=f(x ; \theta, q)=q^{x^{\theta}}-q^{(x+1)^{\theta}}, \quad x \in \mathbb{N}^{*}, \\
& h_{F}(x)=\frac{q^{x^{\theta}}}{q^{(x+1)^{\theta}}}-1, \quad x \in \mathbb{N}^{*},
\end{aligned}
$$

and

$$
r_{F}(x)=\frac{q^{x^{\theta}}-q^{(x+1)^{\theta}}}{1-q^{(x+1)^{\theta}}}, \quad x \in \mathbb{N} *
$$


where $\theta>0$ and $q \in(0,1), \mu \in \mathbb{R}$ are parameters. The reason for using (1.1.3) and (1.1.3)* is that for all of the distributions, except one, we will be using the hazard function.

(b) The cdf, pmf, hf and rhf of DWII distribution are given, respectively, by

$$
\begin{aligned}
& F(x)=F(x ; \beta, c)=1-\prod_{i=1}^{x}\left(1-c i^{\beta-1}\right), \quad x \in \mathbb{N}, \\
& f(x)=f(x ; \beta, c) \\
& \quad=\prod_{i=1}^{x-1}\left(1-c i^{\beta-1}\right)-\prod_{i=1}^{x}\left(1-c i^{\beta-1}\right), x \in \mathbb{N}, \\
& h_{F}(x)=\frac{1}{1-c x^{\beta-1}}-1=\frac{c x^{\beta-1}}{1-c x^{\beta-1}}, \quad x \in \mathbb{N},
\end{aligned}
$$

and

$$
r_{F}(x)=\frac{c x^{\beta-1} \Pi_{i=1}^{x-1}\left(1-h_{F}(i)\right)}{1-\Pi_{i=1}^{x}\left(1-h_{F}(i)\right)}, \quad x \in \mathbb{N},
$$

where $\beta \leq 1,0<c \leq 1$ are parameters.

Remark 1.1. The authors consider two case $\beta \leq 1$ and $\beta>1$. The case $\beta \leq 1$ is considered here since in this case, $x \in \mathbb{N}$ which seems compatible with the other six distributions taken up in this work. The formulas given above are slightly different from those of the authors.

(c) The cdf , pmf, hf and rhf of DWIII distribution are given, respectively, by

$$
\begin{aligned}
& F(x)=F(x ; \alpha, \theta)=1-e^{-\alpha \sum_{j=1}^{x+1} j^{\theta}}, \quad x \in \mathbb{N}, \\
& f(x)=f(x ; \alpha, \theta)=e^{-\alpha \sum_{j=1}^{x} j^{\theta}}-e^{-\alpha \sum_{j=1}^{x+1} j^{\theta}}, x \in \mathbb{N}, \\
& h_{F}(x)=e^{\alpha(x+1)^{\theta}}-1, \quad x \in \mathbb{N},
\end{aligned}
$$

and

$$
r_{F}(x)=\frac{e^{-\alpha \sum_{j=1}^{x} j^{\theta}}-e^{-\alpha \sum_{j=1}^{x+1} j^{\theta}}}{1-e^{-\alpha \sum_{j=1}^{x+1} j^{\theta}}}, \quad x \in \mathbb{N},
$$

where $\alpha>0$ and $\theta \in \mathbb{R}$ are parameters.

(d) The cdf, pmf, hf and rhf of DIW distribution are given, respectively, by

$$
\begin{aligned}
& F(x)=F(x ; \theta, q)=q^{x^{-\theta}}, \quad x \in \mathbb{N}, \\
& f(x)=f(x ; \theta, q)=\left\{\begin{array}{l}
q, \\
q^{x^{-\theta}}-q^{(x-1)^{-\theta}},
\end{array}, x=2,3, \ldots,\right. \\
& h_{F}(x)=\frac{q^{x^{-\theta}}-q^{(x-1)^{-\theta}}}{1-q^{x^{-\theta}}}-1, \quad x \in \mathbb{N},
\end{aligned}
$$

and

$$
r_{F}(x)=1-\frac{q^{(x-1)^{-\theta}}}{q^{x^{-\theta}}}, \quad x \in \mathbb{N},
$$

where $\theta>0$ and $q \in(0,1)$ are parameters.

(e) The cdf, pmf,hf and rhf of DMW distribution are given, respectively, by

$$
\begin{aligned}
& F(x)=F(x ; \theta, c, q)=1-q^{(x+1)^{\theta} c^{x+1}}, x \in \mathbb{N}^{*}, \\
& f(x)=f(x ; \theta, c, q)=q^{x^{\theta} c^{x}}-q^{(x+1)^{\theta} c^{x+1}}, x \in \mathbb{N}^{*}, \\
& h_{F}(x)=\frac{q^{x^{\theta} c^{x}}}{q^{(x+1)^{\theta} c^{x+1}}}-1, \quad x \in \mathbb{N}^{*},
\end{aligned}
$$

and

$$
r_{F}(x)=\frac{q^{x^{\theta} c^{x}}-q^{(x+1)^{\theta} c^{x+1}}}{1-q^{(x+1)^{\theta} c^{x+1}}}, \quad x \in \mathbb{N}^{*},
$$

where $\theta>0, c>0$ and $q \in(0,1)$ are parameters.

(f) The cdf, pmf,hf and rhf of DAW distribution are given, respectively, by

$$
\begin{aligned}
& F(x)=F\left(x ; \theta, \gamma, q_{1}, q_{2}\right)=1-q_{1}^{(x+1)^{\theta}} q_{2}^{(x+1)^{\gamma}}, x \in \mathbb{N}^{*} \\
& f(x)=f\left(x ; \theta, \gamma, q_{1}, q_{2}\right)=q_{1}^{x^{\theta}} q_{2}^{x^{\gamma}}-q_{1}^{(x+1)^{\theta}} q_{2}^{(x+1)^{\gamma}}, \\
& h_{F}(x)=\frac{q_{1}^{x^{\theta}} q_{2}^{x^{\gamma}}}{q_{1}^{(x+1)^{\theta}} q_{2}^{(x+1)^{\gamma}}}-1, \quad x \in \mathbb{N}^{*},
\end{aligned}
$$

and

$$
r_{F}(x)=\frac{q_{1}^{x^{\theta}} q_{2}^{x^{\gamma}}-q_{1}^{(x+1)^{\theta}} q_{2}^{(x+1)^{\gamma}}}{1-q_{1}^{(x+1)^{\theta}} q_{2}^{(x+1)^{\gamma}}}, \quad x \in \mathbb{N}^{*},
$$

where $\theta>0, \gamma>0, q_{1} \in(0,1)$ and $q_{2} \in(0,1)$ are parameters.

$(g)$ The cdf , pmf, hf and rhf of DRMW distribution are given, respectively, by 


$$
\begin{aligned}
& F(x)=F(x ; b, c, q)=1-q^{\sqrt{x+1}\left(1+b c^{x+1}\right)}, x \in \mathbb{N}^{*}, \\
& f(x)=f(x ; b, c, q)=q^{\sqrt{x}\left(1+b c^{x}\right)}-q^{\sqrt{x+1}\left(1+b c^{x+1}\right)}, x \in \mathbb{N}^{*}, \\
& h_{F}(x)=\frac{q^{\sqrt{x}\left(1+b c^{x}\right)}}{q^{\sqrt{x+1}\left(1+b c^{x+1}\right)}-1, \quad x \in \mathbb{N}^{*},}
\end{aligned}
$$

and

$$
r_{F}(x)=\frac{q^{\sqrt{x}\left(1+b c^{x}\right)}-q^{\sqrt{x+1}\left(1+b c^{x+1}\right)}}{1-q^{\sqrt{x+1}\left(1+b c^{x+1}\right)}}, \quad x \in \mathbb{N}^{*},
$$

where $b>0, c \geq 1$ and $q \in(0,1)$ are parameters.

\section{Characterizations Based on Conditional Expectation}

In this section we present our characterizations of all the distributions listed in the Introduction in terms of the truncated moments of certain functions of the random variables. The choice of each function depends on the form of the pmf. We devote a sub-section to each one of the seven distributions. Most of the proofs follow the same scheme, we will give all of them for the sake of completeness.

\subsection{Discrete Weibull (DWI) Distribution}

Nakagawa and Osaki (1975) proposed the first analogue of two-parameter continuous Weibull distribution.

Proposition 2.1.1. Let $X: \Omega \rightarrow \mathbb{N}^{*}$ be a random variable. The pmf of $X$ is (1.1.2) if and only if

$$
E\left\{\left[q^{X^{\theta}}+q^{(X+1)^{\theta}}\right] \mid X>k\right\}=q^{(k+1)^{\theta}}, \quad k \in \mathbb{N}^{*},
$$

Proof. If $X$ has pmf (1.1.2), then for $k \in \mathbb{N}^{*}$, the left-hand side of (2.1.1) will be

$$
(1-F(k))^{-1} \sum_{x=k+1}^{\infty}\left[q^{2 x^{\theta}}-q^{2(x+1)^{\theta}}\right]=q^{-(k+1)^{\theta}} q^{-(k+1)^{\theta}}=q^{(k+1)^{\theta}} .
$$

Conversely, if (2.1.1) holds, then

$$
\begin{aligned}
& \sum_{x=k+1}^{\infty}\left\{\left[q^{x^{\theta}}+q^{(x+1)^{\theta}}\right] f(x)\right\}=(1-F(k)) q^{(k+1)^{\theta}} \\
& =\{(1-F(k+1)+f(k+1))\} q^{(k+1)^{\theta}} .
\end{aligned}
$$

From (2.1.2), we also have

$$
\begin{aligned}
& \sum_{x=k+2}^{\infty}\left\{\left[q^{x^{\theta}}+q^{(x+1)^{\theta}}\right] f(x)\right\} \\
& =(1-F(k+1)) q^{(k+2)^{\theta}} .
\end{aligned}
$$

Now, subtracting (2.1.3) from (2.1.2), yields

$$
\begin{aligned}
& {\left[q^{(k+1)^{\theta}}+q^{(k+2)^{\theta}}\right] f(k+1)} \\
& =(1-F(k+1))\left\{q^{(k+1)^{\theta}}-q^{(k+2)^{\theta}}\right\} \\
& +f(k+1) q^{(k+1)^{\theta}} .
\end{aligned}
$$

From the above equality, we have

$$
h_{F}(k+1)=\frac{f(k+1)}{(1-F(k+1))}=\frac{q^{(k+1)^{\theta}}-q^{(k+2)^{\theta}}}{q^{(k+2)^{\theta}}}=\frac{q^{(k+1)^{\theta}}}{q^{(k+2)^{\theta}}}-1,
$$

which, in view of (1.1.3), implies that $X$ has pmf (1.1.2).

\subsection{Discrete Weibull (DWII) Distribution}

As mentioned by Almalki and Nadarajah (2014), Stein and Dattero (1984) introduced a discrete Weibull distribution called type II discrete Weibull distribution by taking the lifetimes as the integer part of the continuous Weibull distribution.

Proposition 2.2.1. Let $X: \Omega \rightarrow \mathbb{N}^{*}$ be a random variable. The pmf of $X$ is (1.1.5) if and only if

$$
\begin{aligned}
& E\left\{\left[\Pi_{i=1}^{X-1}\left(1-c i^{\beta-1}\right)+\Pi_{i=1}^{X}\left(1-c i^{\beta-1}\right)\right] \mid X>k\right\} \\
& =\Pi_{i=1}^{k}\left(1-c i^{\beta-1}\right), \quad x \in \mathbb{N},
\end{aligned}
$$

Proof. If $X$ has pmf (1.1.5), then for $k \in \mathbb{N}$, the left-hand side of (2.2.1) will be

$$
\begin{aligned}
& (1-F(k))^{-1} \sum_{x=k+1}^{\infty}\left[\Pi_{i=1}^{x-1}\left(1-c i^{\beta-1}\right)^{2}-\Pi_{i=1}^{x}\left(1-c i^{\beta-1}\right)^{2}\right] \\
& =\Pi_{i=1}^{k}\left(1-c i^{\beta-1}\right)^{-1} \Pi_{i=1}^{k}\left(1-c i^{\beta-1}\right)^{2}=\Pi_{i=1}^{k}\left(1-c i^{\beta-1}\right) .
\end{aligned}
$$

Similarly, for $k<0$ and $k \in \mathbb{Z}$, we obtain the right hand side of (2.2.1).

Conversely, if (2.2.1) holds, then

$$
\sum_{x=k+1}^{\infty}\left\{\left[\Pi_{i=1}^{x-1}\left(1-c i^{\beta-1}\right)+\Pi_{i=1}^{x}\left(1-c i^{\beta-1}\right)\right] f(x)\right\}
$$




$$
=\{(1-F(k+1))+f(k+1)\} \Pi_{i=1}^{k}\left(1-c i^{\beta-1}\right) .
$$

From (2.2.2), we also have

$$
\begin{aligned}
& \sum_{x=k+2}^{\infty}\left\{\left[\Pi_{i=1}^{x-1}\left(1-c i^{\beta-1}\right)+\Pi_{i=1}^{x}\left(1-c i^{\beta-1}\right)\right] f(x)\right\} \\
& =(1-F(k+1)) \Pi_{i=1}^{k+1}\left(1-c i^{\beta-1}\right) .
\end{aligned}
$$

Subtracting (2.2.3) from (2.2.2) and rearranging terms, yields

$$
\begin{aligned}
& \left\{\Pi_{i=1}^{k}\left(1-c i^{\beta-1}\right)+\Pi_{i=1}^{x+1}\left(1-c i^{\beta-1}\right)\right\} f(k+1) \\
& =\left\{\Pi_{i=1}^{k}\left(1-c i^{\beta-1}\right)-\Pi_{i=1}^{k+1}\left(1-c i^{\beta-1}\right)\right\}(1-F(k+1))+ \\
& \prod_{i=1}^{k}\left(1-c i^{\beta-1}\right) f(k+1)
\end{aligned}
$$

or

$$
\left\{\Pi_{i=1}^{k+1}\left(1-c i^{\beta-1}\right)\right\} f(k+1)=\left\{\Pi_{i=1}^{k}\left(1-c i^{\beta-1}\right)-\Pi_{i=1}^{k+1}\left(1-c i^{\beta-1}\right)\right\}(1-F(k+1)) .
$$

From the above equality, after some manipulations, we have

$$
\begin{aligned}
& h_{F}(k+1)=\frac{f(k+1)}{1-F(k+1)} \\
& =\frac{\left\{\Pi_{i=1}^{k}\left(1-c i^{\beta-1}\right)-\Pi_{i=1}^{k+1}\left(1-c i i^{\beta-1}\right)\right\}}{\Pi_{i=1}^{k+1}\left(1-c i^{\beta-1}\right)} \\
& =\frac{1}{1-c x^{\beta-1}}-1,
\end{aligned}
$$

which in view of (1.1.6), implies that $X$ has mpf (1.1.5).

\subsection{Discrete Weibull (DWIII) Distribution}

Again as pointed Almalki and Nadarajah (2014), the third discrete version of the Weibull distribution was introduced by Padgett and Spurrier (1985). It exhibit increasing, decreasing and constant hazard function.

Proposition 2.3.1 . Let $X: \Omega \rightarrow \mathbb{N}^{*}$ be a random variable. The pmf of $X$ is (1.1.8) if and only if

$$
E\left\{\left[e^{-\alpha \sum_{j=1}^{X} j^{\theta}}+e^{-\alpha \sum_{j=1}^{X+1} j^{\theta}}\right] \mid X>k\right\}=e^{-\alpha \sum_{j=1}^{k+1} j^{\theta}}
$$

Proof. We take $-1 \leq \theta<0$ or $\theta>0$ to make sure that the infinite series is convergent. If $X$ has pmf (1.1.8), then for $k \in \mathbb{N}^{*}$, the left-hand side of (2.3.1) will be

$$
\begin{aligned}
& (1-F(k))^{-1} \sum_{x=k+1}^{\infty}\left[e^{-2 \alpha \sum_{j=1}^{X} j^{\theta}}-e^{-2 \alpha \sum_{j=1}^{X+1} j^{\theta}}\right] \\
& =e^{\alpha \sum_{j=1}^{X} j^{\theta}} e^{-2 \alpha \sum_{j=1}^{X} j^{\theta}}=e^{-\alpha \sum_{j=1}^{k+1} j^{\theta}} .
\end{aligned}
$$

Conversely, if (2.3.1) holds, then

$$
\begin{aligned}
& \sum_{x=k+1}^{\infty}\left\{\left[e^{-\alpha \sum_{j=1}^{x} j^{\theta}}+e^{-\alpha \sum_{j=1}^{x+1} j^{\theta}}\right] f(x)\right\} \\
& =\{(1-F(k+1)+f(k+1))\} e^{-\alpha \sum_{j=1}^{k+1} j^{\theta}} .
\end{aligned}
$$

From (2.3.2), we also have

$$
\begin{aligned}
& \sum_{x=k+2}^{\infty}\left\{\left[e^{-\alpha \sum_{j=1}^{x} j^{\theta}}+e^{-\alpha \sum_{j=1}^{x+1} j^{\theta}}\right] f(x)\right\} \\
& =\{1-F(k+1)\} e^{-\alpha \sum_{j=1}^{k+2} j^{\theta}} .
\end{aligned}
$$

Now, subtracting (2.3.3) from (2.3.2), yields

$$
e^{-\alpha \sum_{j=1}^{k+2} j^{\theta}} f(k+1)=(1-F(k+1))\left\{e^{-\alpha \sum_{j=1}^{k+1} j^{\theta}}-e^{-\alpha \sum_{j=1}^{k+2} j^{\theta}}\right\}
$$

From the above equality, we have

$$
\frac{f(k+1)}{(1-F(k+1))}=\frac{e^{-\alpha \sum_{j=1}^{k+1} j^{\theta}}-e^{-\alpha \sum_{j=1}^{k+2} j^{\theta}}}{e^{-\alpha \sum_{j=1}^{k+2} j^{\theta}}}=e^{\alpha(k+2)^{\theta}}-1,
$$

which, in view of (1.1.9), implies that $X$ has pmf (1.1.8).

Remark 2.3.1. Proposition 2.3 .1 can be stated for the case $f(x)=f(x ; \theta, \alpha)=\theta^{x^{\alpha}}-\theta^{(x+1)^{\alpha}}, \alpha>0, x \in$ $\mathbb{N}^{*}$, which is also called discrete Weibull, due to Nakagawa et al. (1975).

\subsection{Discrete Inverse Weibull (DIW) Distribution}

Jazi et al. (2010) proposed the discrete inverse Weibull distribution with two parameters by considering certain property of the continuous inverse Weibull distribution. 
Proposition 2.4.1 . Let $X: \Omega \rightarrow \mathbb{N}$ be a random variable. The pmf of $X$ is (1.1.11) if and only if

$$
E\left\{\left[q^{X^{-\theta}}+q^{(X-1)^{-\theta}}\right] \mid X \leq k\right\}=q^{k^{-\theta}} .
$$

Proof. If $X$ has pmf (1.1.11), then for $k \in \mathbb{N}^{*}$, the left-hand side of (2.4.1) will be

$$
(F(k))^{-1} \sum_{x=1}^{k}\left[q^{2 x^{-\theta}}-q^{2(x-1)^{-\theta}}\right]=q^{-k^{-\theta}} q^{2 k^{-\theta}}=q^{k^{-\theta}} .
$$

Conversely, if (2.4.1) holds, then

$$
\sum_{x=1}^{k}\left\{\left[q^{x^{-\theta}}+q^{(x-1)^{-\theta}}\right] f(x)\right\}=F(k) q^{k^{-\theta}} .
$$

From (2.4.2), we also have

$$
\begin{aligned}
& \sum_{x=1}^{k-1}\left\{\left[q^{x^{-\theta}}+q^{(x-1)^{-\theta}}\right] f(x)\right\} \\
& =\{F(k)-f(k)\} q^{(k-1)^{-\theta}} .
\end{aligned}
$$

Subtracting (2.4.3) from (2.4.2) and rearranging terms, yields

$$
\begin{aligned}
& \left\{\left[q^{k^{-\theta}}+q^{(k-1)^{-\theta}}\right]\right\} f(k) \\
& =F(k)\left\{q^{k^{-\theta}}+q^{(k-1)^{-\theta}}\right\}+f(k) q^{(k-1)^{-\theta}} .
\end{aligned}
$$

From the above equality, we have

$$
r_{F}(k)=\frac{f(k)}{F(k)}=1-\frac{q^{(k-1)^{-\theta}}}{q^{k^{-\theta}}},
$$

which in view of (1.1.12), implies that $X$ has mpf (1.1.11).

\subsection{Discrete Modified Weibull (DMW) Distribution}

This distribution was proposed by Nooghabi et al. (2011) which is the discrete analogue of the modified Weibull distribution of Lai et al. (2003).

Proposition 2.5.1 . Let $X: \Omega \rightarrow \mathbb{N}^{*}$ be a random variable. The pmf of $X$ is (1.1.14) if and only if

$$
E\left\{\left[q^{X^{\theta} c^{X}}+q^{(X+1)^{\theta} c^{X+1}}\right] \mid X>k\right\}=q^{(k+1)^{\theta} c^{k+1}} .
$$

Proof. If $X$ has pmf (1.1.14), then for $k \in \mathbb{N}^{*}$, the left-hand side of (2.5.1) will be

$$
\begin{aligned}
& (1-F(k))^{-1} \sum_{x=k+1}^{\infty}\left[q^{2 x^{\theta} c^{x}}-q^{2(x+1)^{\theta} c^{x+1}}\right] \\
& =q^{-(k+1)^{\theta} c^{k+1}} q^{2(k+1)^{\theta} c^{k+1}}=q^{(k+1)^{\theta} c^{k+1}} .
\end{aligned}
$$

Conversely, if (2.5.1) holds, then

$$
\begin{aligned}
& \sum_{x=k+1}^{\infty}\left\{\left[q^{x^{\theta} c^{x}}+q^{(x+1)^{\theta} c^{x+1}}\right] f(x)\right\} \\
& =\{(1-F(k+1))+f(k+1)\} q^{(k+1)^{\theta} c^{k+1}} .
\end{aligned}
$$

From (2.5.2), we also have

$$
\begin{aligned}
& \sum_{x=k+2}^{\infty}\left\{\left[q^{x^{\theta} c^{x}}+q^{(x+1)^{\theta} c^{x+1}}\right] f(x)\right\} \\
& =(1-F(k+1)) q^{(k+2)^{\theta} c^{k+2}} .
\end{aligned}
$$

Subtracting (2.5.3) from (2.5.2) and rearranging terms, yields

$$
\begin{aligned}
& \left\{q^{(k+2)^{\theta} c^{k+2}}\right\} f(k+1) \\
& =\left\{\left[q^{(k+1)^{\theta} c^{k+1}}-q^{(k+2)^{\theta} c^{k+2}}\right]\right\}(1-F(k+1)) .
\end{aligned}
$$

From the above equality, after some manipulations, we have

$$
\frac{f(k+1)}{1-F(k+1)}=\frac{q^{(k+1) \theta} c^{k+1}}{q^{(k+2)^{\theta} c^{k+2}}}-1,
$$

which in view of (1.1.15), implies that $X$ has mpf (1.1.14).

\subsection{Discrete Additive Weibull (DAW) Distribution}

Bebbington et al. (2012) proposed the four-parameter discrete additive Weibull distribution.

Proposition 2.6.1 . Let $X: \Omega \rightarrow \mathbb{N}^{*}$ be a random variable. The pmf of $X$ is (1.1.16) if and only if

$$
E\left\{\left[q_{1}^{X^{\theta}} q_{2}^{X^{\gamma}}+q_{1}^{(X+1)^{\theta}} q_{2}^{(X+1)^{\gamma}}\right] \mid X>k\right\}=q_{1}^{(k+1)^{\theta}} q_{2}^{(k+1)^{\gamma}} .
$$

Proof. If $X$ has pmf (1.1.16), then for $k \in \mathbb{N}^{*}$, the left-hand side of (2.6.1) will be

$$
(1-F(k))^{-1} \sum_{x=k+1}^{\infty}\left[q_{1}^{2 x^{\theta}} q_{2}^{2 x^{\gamma}}-q_{1}^{2(x+1)^{\theta}} q_{2}^{2(x+1)^{\gamma}}\right]
$$




$$
=q_{1}^{-(k+1)^{\theta}} q_{2}^{-(k+1)^{\gamma}} q_{1}^{2(k+1)^{\theta}} q_{2}^{2(k+1)^{\gamma}}=q_{1}^{(k+1)^{\theta}} q_{2}^{(k+1)^{\gamma}} .
$$

Conversely, if (2.6.1) holds, then

$$
\begin{aligned}
& \sum_{x=k+1}^{\infty}\left\{\left[q_{1}^{x^{\theta}} q_{2}^{x^{\gamma}}+q_{1}^{(x+1)^{\theta}} q_{2}^{(x+1)^{\gamma}}\right] f(x)\right\} \\
& =\{(1-F(k+1))+f(k+1)\} q_{1}^{(k+1)^{\theta}} q_{2}^{(k+1)^{\gamma}} .
\end{aligned}
$$

From (2.6.2), we also have

$$
\begin{aligned}
& \sum_{x=k+2}^{\infty}\left\{\left[q_{1}^{x^{\theta}} q_{2}^{x^{\gamma}}+q_{1}^{(x+1)^{\theta}} q_{2}^{(x+1)^{\gamma}}\right] f(x)\right\} \\
& =(1-F(k+1)) q_{1}^{(k+2)^{\theta}} q_{2}^{(k+2)^{\gamma}} .
\end{aligned}
$$

Subtracting (2.6.3) from (2.6.2), yields

$$
\begin{aligned}
& \left\{q_{1}^{(k+2)^{\theta}} q_{2}^{(k+2)^{\gamma}}\right\} f(k+1) \\
& =\left\{q_{1}^{(k+1)^{\theta}} q_{2}^{(k+1)^{\gamma}}-q_{1}^{(k+2)^{\theta}} q_{2}^{(k+2)^{\gamma}}\right\}(1-F(k+1)) .
\end{aligned}
$$

From the above equality, after some manipulations, we have

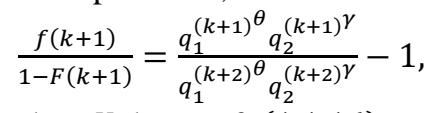

which in view of (1.1.18), implies that $X$ has mpf (1.1.16).

\subsection{Discrete Reduced Modified Weibull (DRMW) Distribution}

Almalki and Nadarajah (2014) introduced a three-parameter discrete analogue of Almalki's reduced modified Weibull distribution, (2018).

Proposition 2.7.1 . Let $X: \Omega \rightarrow \mathbb{N}^{*}$ be a random variable. The pmf of $X$ is (1.1.20) if and only if

$$
\begin{aligned}
& E\left\{\left[q^{\sqrt{X}\left(1+b c^{X}\right)}+q^{\sqrt{X+1}\left(1+b c^{X+1}\right)}\right] \mid X>k\right\} \\
& =q^{\sqrt{k+1}\left(1+b c^{k+1}\right)} .
\end{aligned}
$$

Proof. If $X$ has pmf (1.1.20), then for $k \in \mathbb{N}^{*}$, the left-hand side of (2.7.1) will be

$$
\begin{aligned}
& (1-F(k))^{-1} \sum_{x=k+1}^{\infty}\left[q^{2 \sqrt{x}\left(1+b c^{x}\right)}-q^{2 \sqrt{x+1}\left(1+b c^{x+1}\right)}\right] \\
& =q^{-\sqrt{k+1}\left(1+b c^{k+1}\right)} q^{2 \sqrt{k+1}\left(1+b c^{k+1}\right)}=q^{\sqrt{k+1}\left(1+b c^{k+1}\right) .}
\end{aligned}
$$

Conversely, if (2.7.1) holds, then

$$
\begin{aligned}
& \sum_{x=k+1}^{\infty}\left\{\left[q^{\sqrt{x}\left(1+b c^{x}\right)}+q^{\sqrt{x+1}\left(1+b c^{x+1}\right)}\right] f(x)\right\} \\
& =\{(1-F(k+1))+f(k+1)\} q^{\sqrt{k+1}\left(1+b c^{k+1}\right)} .
\end{aligned}
$$

From (2.7.2), we also have

$$
\begin{aligned}
& \sum_{x=k+2}^{\infty}\left\{\left[q^{\sqrt{x}\left(1+b c^{x}\right)}+q^{\sqrt{x+1}\left(1+b c^{x+1}\right)}\right] f(x)\right\} \\
& =(1-F(k+1)) q^{\sqrt{k+2}\left(1+b c^{k+2}\right)} .
\end{aligned}
$$

Subtracting (2.7.3) from (2.7.2), yields

$$
\begin{aligned}
& \left\{q^{\sqrt{k+2}\left(1+b c^{k+2}\right)}\right\} f(k+1) \\
& =\left\{q^{\sqrt{k+1}\left(1+b c^{k+1}\right)}-q^{\sqrt{k+2}\left(1+b c^{k+2}\right)}\right\}(1-F(k+1)) .
\end{aligned}
$$

From the above equality, after some manipulations, we have

$$
\frac{f(k+1)}{1-F(k+1)}=\frac{q^{\sqrt{k+1}\left(1+b c^{k+1}\right)}}{q^{\sqrt{k+2}\left(1+b c^{k+2}\right)}}-1,
$$

which in view of (1.1.21), implies that $X$ has mpf (1.1.20).

\section{Characterizations of distributions based on hazard function}

This section consists of 6 sub-sections devoted to 6 of the 7 distributions listed in the Introduction. The characterizations presented here are in terms of the hazard function. Most of the proofs follow the same scheme and some can be omitted. We, however, give all the proofs and the reader may choose to skip the proofs. Due to the fact that these 6 distributions form a subset of those mentioned in Section 2, we will not repeat the statements given at the beginning of the corresponding sections for them in this section. 


\subsection{Discrete Weibull (DWI) Distribution}

Proposition 3.1.1. Let $X: \Omega \rightarrow \mathbb{N}^{*}$ be a random variable. The pmf of $X$ is (1.1.2) if and only if its hazard function satisfies the difference equation

$$
h_{F}(k+1)-h_{F}(k)=\frac{q^{(k+1)^{\theta}}}{q^{(k+2)^{\theta}}}-\frac{q^{k^{\theta}}}{q^{(k+1)^{\theta}}}, k \in \mathbb{N}^{*},
$$

with the initial condition $h_{F}(0)=\frac{1-q}{q}$. we have

Proof. If $X$ has pmf (1.1.2), then clearly (3.1.1) holds. Now, if (3.1.1) holds, then for every $x \in \mathbb{N}$,

or

$$
\sum_{k=0}^{x-1}\left\{h_{F}(k+1)-h_{F}(k)\right\}=\sum_{k=0}^{x-1}\left\{\frac{q^{(k+1)^{\theta}}}{q^{(k+2)^{\theta}}}-\frac{q^{k^{\theta}}}{q^{(k+1)^{\theta}}}\right\},
$$

or, in view of the initial condition $h_{F}(0)=\frac{1-q}{q}$,

$$
h_{F}(x)-h_{F}(0)=-\frac{1}{q}+\frac{q^{x^{\theta}}}{q^{(x+1)^{\theta}}}
$$

\subsection{Discrete Weibull (DWII) Distribution}

$$
h_{F}(x)=\frac{q^{x^{\theta}}}{q^{(x+1)^{\theta}}}-1, \quad x \in \mathbb{N}^{*} .
$$

Proposition 3.2.1. Let $X: \Omega \rightarrow \mathbb{N}$ be a random variable. The pmf of $X$ is (1.1.5) if and only if its hazard function satisfies the difference equation

$$
h_{F}(k+1)-h_{F}(k)=\frac{1}{1-c(k+1)^{\beta-1}}-\frac{1}{1-c k^{\beta-1}}, \quad k \in \mathbb{N},
$$

with the initial condition $h_{F}(1)=\frac{c}{1-c}$.

we have

Proof. If $X$ has pmf (1.1.5), then clearly (3.2.1) holds. Now, if (3.2.1) holds, then for every $x \in \mathbb{N}$,

or

$$
\sum_{k=1}^{x-1}\left\{h_{F}(k+1)-h_{F}(k)\right\}=\sum_{k=1}^{x-1}\left[\frac{1}{1-c(k+1)^{\beta-1}}-\frac{1}{1-c k^{\beta-1}}\right],
$$

or, in view of the initial condition $h_{F}(1)=\frac{c}{1-c}$,

$$
h_{F}(x)-h_{F}(1)=-\frac{1}{1-c}+\frac{1}{1-c x^{\beta-1}},
$$

\subsection{Discrete Weibull (DWIII) Distribution}

$$
h_{F}(x)=\frac{1}{1-c x^{\beta-1}}-1, \quad x \in \mathbb{N} \text {. }
$$

Proposition 3.3.1. Let $X: \Omega \rightarrow \mathbb{N}^{*}$ be a random variable. The pmf of $X$ is (1.1.8) if and only if its hazard function satisfies the difference equation

$$
h_{F}(k+1)-h_{F}(k)=e^{\alpha(k+2)^{\theta}}-e^{\alpha(k+1)^{\theta}}, \quad k \in \mathbb{N}^{*},
$$

with the initial condition $h_{F}(0)=e^{\alpha}-1$. we have

Proof. If $X$ has pmf (1.1.8), then clearly (3.3.1) holds. Now, if (3.3.1) holds, then for every $x \in \mathbb{N}$,

or

$$
\sum_{k=0}^{x-1}\left\{h_{F}(k+1)-h_{F}(k)\right\}=\sum_{k=0}^{x-1}\left\{e^{\alpha(k+2)^{\theta}}-e^{\alpha(k+1)^{\theta}}\right\},
$$

or, in view of the initial condition $h_{F}(0)=e^{\alpha}-1$,

$$
h_{F}(x)-h_{F}(0)=-e^{\alpha}+e^{\alpha(x+1)^{\theta}},
$$

$$
h_{F}(x)=e^{\alpha(x+1)^{\theta}}-1, \quad x \in \mathbb{N} .
$$

\subsection{Discrete Modified Weibull (DMW) Distribution}

Proposition 3.4.1. Let $X: \Omega \rightarrow \mathbb{N}^{*}$ be a random variable. The pmf of $X$ is (1.1.14) if and only if its hazard function satisfies the difference equation

$$
h_{F}(k+1)-h_{F}(k)=\frac{q^{(k+1)^{\theta} c^{k+1}}}{q^{(k+2)^{\theta} c^{k+2}}}-\frac{q^{k^{\theta} c^{k}}}{q^{(k+1)^{\theta} c^{k+1}}}, k \in \mathbb{N}
$$


with the initial condition $h_{F}(0)=\frac{1}{q^{c}}-1$.

Proof. If $X$ has pmf (1.1.14), then clearly (3.4.1) holds. Now, if (3.4.1) holds, then for every $x \in \mathbb{N}$ , we have

or

$$
\sum_{k=0}^{x-1}\left\{h_{F}(k+1)-h_{F}(k)\right\}=\sum_{k=0}^{x-1}\left\{\frac{q^{(k+1)^{\theta} c^{k+1}}}{q^{(k+2)^{\theta} c^{k+2}}}-\frac{q^{k^{\theta} c^{k}}}{q^{(k+1)^{\theta} c^{k+1}}}\right\}
$$

or

$$
h_{F}(x)-h_{F}(0)=-\frac{1}{q^{c}}+\frac{q^{x^{\theta} c^{k}}}{q^{(x+1)^{\theta} c^{k+1}}}, \quad x \in \mathbb{N}^{*},
$$

$$
h_{F}(x)=\frac{q^{x^{\theta} c^{k}}}{q^{(x+1)^{\theta} c^{k+1}}}-1, \quad x \in \mathbb{N}^{*}
$$

\subsection{Discrete Additive Weibull (DAW) Distribution}

Proposition 3.5.1. Let $X: \Omega \rightarrow \mathbb{N}^{*}$ be a random variable. The pmf of $X$ is (1.1.17) if and only if its hazard function satisfies the difference equation

$$
h_{F}(k+1)-h_{F}(k)=\frac{q_{1}^{(k+1)^{\theta}} q_{2}^{(k+1)^{\gamma}}}{q_{1}^{(k+2)^{\theta}} q_{2}^{(k+2)^{\gamma}}}-\frac{q_{1}^{k^{\theta}} q_{2}^{k \gamma}}{q_{1}^{(k+1)^{\theta}} q_{2}^{(k+1)^{\gamma}}}, k \in \mathbb{N}^{*},
$$

with the initial condition $h_{F}(0)=\frac{1}{q_{1} q_{2}}-1$. , we have

or

Proof. If $X$ has pmf (1.1.17), then clearly (3.5.1) holds. Now, if (3.5.1) holds, then for every $x \in \mathbb{N}$

or

$$
\sum_{k=0}^{x-1}\left\{h_{F}(k+1)-h_{F}(k)\right\}=\sum_{k=0}^{x-1}\left\{\frac{q_{1}^{(k+1)^{\theta}} q_{2}^{(k+1)^{\gamma}}}{q_{1}^{(k+2)^{\theta}} q_{2}^{(k+2)^{\gamma}}}-\frac{q_{1}^{k^{\theta}} q_{2}^{k^{\gamma}}}{q_{1}^{(k+1)^{\theta}} q_{2}^{(k+1)^{\gamma}}}\right\}
$$

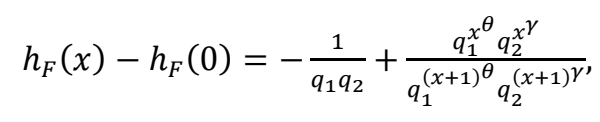

$$
h_{F}(x)=\frac{q_{1}^{x^{\theta}} q_{2}^{x^{\gamma}}}{q_{1}^{(x+1)^{\theta}} q_{2}^{(x+1)^{\gamma}}}-1, \quad x \in \mathbb{N}^{*}
$$

\subsection{Discrete Reduced Modified Weibull (DRMW) Distribution}

Proposition 3.6.1. Let $X: \Omega \rightarrow \mathbb{N}^{*}$ be a random variable. The pmf of $X$ is (1.1.20) if and only if its hazard function satisfies the difference equation

$$
h_{F}(k+1)-h_{F}(k)=\frac{q^{\sqrt{k+1}\left(1+b c^{k+1}\right)}}{q^{\sqrt{k+2}\left(1+b c^{k+2}\right)}}-\frac{q^{\sqrt{k}\left(1+b c^{k}\right)}}{q^{\sqrt{k+1}\left(1+b c^{k+1}\right)}}, k \in \mathbb{N}^{*},
$$

with the initial condition $h_{F}(0)=\frac{1}{q^{1+b c}}-1$.

Proof. If $X$ has pmf (1.1.20), then clearly (3.6.1) holds. Now, if (3.6.1) holds, then for every $x \in \mathbb{N}$, we have

or

$$
\sum_{k=0}^{x-1}\left\{h_{F}(k+1)-h_{f}(k)\right\}=\sum_{k=0}^{x-1}\left\{\frac{\left.q^{\sqrt{k+1}\left(1+b c^{k+1}\right.}\right)}{q^{\sqrt{k+2}\left(1+b c^{k+2}\right)}}-\frac{q^{\sqrt{k}\left(1+b c^{k}\right)}}{q^{\sqrt{k+1}\left(1+b c^{k+1}\right)}}\right\}
$$

or

$$
h_{F}(x)-h_{F}(0)=-\frac{1}{q^{1+b c}}+\frac{q^{\sqrt{x}\left(1+b c^{x}\right)}}{q^{\sqrt{x+1}\left(1+b c^{x+1}\right)}},
$$

$$
h_{F}(x)=\frac{q^{\sqrt{x}\left(1+b c^{x}\right)}}{q^{\sqrt{x+1}\left(1+b c^{x+1}\right)}}-1, \quad x \in \mathbb{N}^{*} .
$$




\section{Characterizations of distributions based on reverse hazard function}

This section is devoted to 1 of the 7 distributions listed in the Introduction. The characterization presented here is in terms of the reverse hazard function. The proofs follow the same scheme, we give the proof for the sake of completeness.

\subsection{Discrete Inverse Weibull (DIW) Distribution}

Proposition 4.1.1. Let $X: \Omega \rightarrow \mathbb{N}$ be a random variable. The pmf of $X$ is (1.1.11) if and only if its reverse hazard function satisfies the difference equation

with initial condition $r_{F}(1)=1-\frac{1}{q}$.

$$
r_{F}(k+1)-r_{F}(k)=\frac{q^{(k-1)^{-\theta}}}{q^{k^{-\theta}}}-\frac{q^{k^{-\theta}}}{q^{(k+1)^{-\theta}}}, k \in \mathbb{N},
$$

Proof. If $X$ has pmf (1.1.11), then clearly (4.1.1) holds. Now, if (4.1.1) holds, then for $x \in \mathbb{N}$, we

have

$$
\sum_{k=1}^{x-1}\left\{r_{F}(k+1)-r_{F}(k)\right\}=\sum_{k=1}^{x-1}\left\{\frac{q^{(k-1)^{-\theta}}}{q^{k^{-\theta}}}-\frac{q^{k^{-\theta}}}{q^{(k+1)^{-\theta}}}\right\}
$$

or

$$
r_{F}(x)-r_{F}(0)=\frac{1}{q}-\frac{q^{(x-1)^{-\theta}}}{q^{k^{x-\theta}}}
$$

or, in view of $r_{F}(1)=1-\frac{1}{q}$, we have

$$
r_{F}(x)=1-\frac{q^{(x-1)^{-\theta}}}{q^{k^{x-\theta}}} .
$$

\section{References}

Almalki, S.J. (2018). A reduced new modified Weibull distribution. Commun. Statist. Theo-Meth, 47(10), 22972313.

Almalki, S.J. and Nadarajah, S. (2014). A new discrete modified Weibull distribution. IEEE Trans Reliab, 63(1), 68-80.

Bebbington, M, Lai, C.D., Wellington, M. and Zitikis, R. (2012). The discrete additive Weibull distribution: a bathtub shaped hazard for discontinuous failure data. Reliab Eng syst Safety, 106, 37-44.

Jazi, M.A., Lai, C.D., Alamatsaz, M.H. (2010). A discrete inverse Weibull distribution and estimation of its parameters. Stat Methodol, 7, 121-132.

Lai, C.M., Xie, M. and Murthy, D.N.P. (2003). A modified Weibull distribution. IEEE Trans Reliab, 52, 33-37.

Nakagawa, T. and Osaki, S. (1975). The discrete Weibull distribution. IEEE Trans Reliab, 24, 300-301.

Nooghabi, M.S., Borzadaran, G.R.M. and Roknabadi, A.H.R. (2011). Discrete modified Weibull distribution. Metron, 69, 207-222.

Padgett, W.J., Spurrier, J.D. (1985). On discrete failure models. IEEE Trans Reliab, 34, 253-256.

Stein, E.W. and Dattero, R.A. (1984). A new discrete Weibull distribution. IEEE Trans Reliab, 33, 196-197. 